\title{
Generalized Similar Frenet Curves
}

\author{
Fatma Ateş*, Seher Kaya, Yusuf Yaylı and F. Nejat Ekmekci
}

(Communicated by Levent KULA)

\begin{abstract}
The paper is devoted to differential geometric invariants determining a Frenet curve in $E^{n}$ up to a direct similarity. These invariants can be presented by the Euclidean curvatures in terms of an arc lengths of the spherical indicatrices. Then, they expressed by focal curvatures of the curve. And then, we give the relationship between curvatures of evolute curve and shape curvatures. Morever, the geometric interpretations of these invariants are given.
\end{abstract}

Keywords: Self-similar curves; Similar curves; Shape curvatures; Focal curvatures.

AMS Subject Classification (2010): Primary: 53A55; 53A04.

*Corresponding author

\section{Introduction}

A similarity of the Euclidean space $E^{n}$ is an automorphism of $E^{n}$ for which the ratio: distance between two arbitrary points to distance between the transformed points is a positive constant. A direct similarity is an orientation preserving similarity.

A few important curves which are associated with Frenet curve in $E^{n}$ such as spherical images, focal curve which consist of centers of the osculating spheres, the evolute curve which is the locus of the centers of the Meusnier spheres, etc. In this work, we investigate invariants of the curve under the direct similarity transformations. For this, we use its spherical images $V_{i}$ which is the $i-t h$ Frenet vector field of the curve in $E^{n}$. Arc length of this curve is preserved under the direct similarity transformation which is called spherical arc length of the curve. The curve is reparametrization according to spherical arc length. Then, we calculated some differential-geometric invariants under the direct similarities which are called shape curvatures.

We express the shape curvatures by using the focal curves which are introduced by Uribe-Vargas. Then, we investigate the relationship between the shape curvatures of the curve and the Frenet curvatures of its evolute curve.

\section{Preliminaries}

In this section, we review some basic concepts on classical differential geometry of space curves in Euclidean $n$-space. For any two vectors $x=\left(x_{1}, x_{2}, \ldots, x_{n}\right)$ and $y=\left(y_{1}, y_{2}, \ldots, y_{n}\right) \in E^{n}, x . y$ as the standard inner product. Let $\alpha: I \subset \mathbb{R} \rightarrow E^{n}$ be a curve with $\dot{\alpha}(t) \neq 0$, where $\dot{\alpha}(t)=\frac{d \alpha}{d t}$. We also denote the norm of $x$ by $\|x\|$. The arc length parametres of curve $\alpha$ is determined such that $\left\|\alpha^{\prime}(s)\right\|=1$, where $\alpha^{\prime}(s)=\frac{d \alpha}{d s}$. Let $V_{1}, V_{2}, \ldots, V_{n}$ be a Frenet moving $n$-frame of the curve $\alpha$. Then the following Frenet-Serret formula holds

$$
\begin{aligned}
V_{1}^{\prime}(s) & =\kappa_{1}(s) V_{2}(s) \\
V_{i}^{\prime}(s) & =-\kappa_{i-1}(s) V_{i-1}(s)+\kappa_{i}(s) V_{i+1}(s) \\
V_{n}^{\prime}(s) & =-\kappa_{n-1}(s) V_{n-1}(s)
\end{aligned}
$$

Received : 09-February-2017, Accepted : 22-June-2017 
where $\kappa_{1}, \kappa_{2}, \ldots, \kappa_{n-1}$ are the curvatures of the curve $\alpha$ at the point $s$.

We will study the differential-geometric invariants of a curve in $E^{n}$ with respect to the group $\operatorname{Sim}^{+}\left(\mathbb{R}^{n}\right)$ of all orientation-preserving similarities of $\mathbb{R}^{n}$. Any such similarity $F: \mathbb{R}^{n} \rightarrow \mathbb{R}^{n}$ is called a direct similarity and can be expressed in the form

$$
F(x)=\lambda A x+b
$$

where $x \in E^{n}$ is an arbitrary point, $A$ is an orthogonal $n \times n$ matrix, $b$ is a translation vector and $\lambda$ is a positive real constant.

We denote the image of the curve $\alpha$ under the direct similarity $F$ by the curve $\bar{\alpha}$, i.e., $\bar{\alpha}=F \circ \alpha$. Then, the curve $\bar{\alpha}$ can be expressed as

$$
F \circ \alpha(t)=F(\alpha(t))=\lambda A \alpha(t)+b .
$$

The arc length functions of the curves $\alpha$ and $\bar{\alpha}$ are

$$
s(t)=\int_{t_{0}}^{t}\left\|\frac{d \alpha(u)}{d u}\right\| d u \quad \text { and } \quad \bar{s}(t)=\int_{t_{0}}^{t}\left\|\frac{d \bar{\alpha}(u)}{d u}\right\| d u=\lambda s(t) .
$$

Let $\left\{\bar{V}_{1}, \bar{V}_{2}, \ldots, \bar{V}_{n}, \bar{\kappa}_{1}, \bar{\kappa}_{2}, \ldots, \bar{\kappa}_{(n-1)}\right\}$ be a Frenet apparatus of the curve $\bar{\alpha}$. Since $\frac{d s}{d \bar{s}}=\frac{1}{\lambda}(=$ const.) the curvatures of the curve $\bar{\alpha}$ are given by

$$
\bar{\kappa}_{i}=\frac{1}{\lambda} \kappa_{i}(s) \quad, \quad i=1,2, \ldots, n-1
$$

\section{The expression of the curve $\alpha$ according to the parameter $\sigma_{i}$}

In this section, we give some characterizations of the curve $\alpha$ by using the arc length parameters of its $V_{i}$ indicatrix curve.

Let $\gamma\left(\sigma_{i}\right)=V_{i}(s)$ be a spherical $V_{i}$ indicatrix curve of the curve $\alpha$ with the arc length parameter $\sigma_{i}$. Then the curve $\alpha$ admits a reparametrization by $\sigma_{i}$

$$
\alpha=\alpha\left(\sigma_{i}\right): I \subset \mathbb{R} \rightarrow E^{n} .
$$

It is clear that

$$
d \sigma_{i}=\sqrt{\left(\kappa_{i-1}(s)\right)^{2}+\left(\kappa_{i}(s)\right)^{2}} d s \quad, \quad \frac{d}{d \sigma_{i}}=\frac{1}{\sqrt{\left(\kappa_{i-1}\right)^{2}+\left(\kappa_{i}\right)^{2}}} \frac{d}{d s} .
$$

The function $d \sigma_{i}=\sqrt{\left(\kappa_{i-1}\right)^{2}+\left(\kappa_{i}\right)^{2}} d s$ is an invariant under the group of the direct similarities of $E^{n}$.

Let $V_{1}, V_{2}, \ldots, V_{n}$ be a Frenet frame vectors along the curve $\alpha$ parameterized by the arc length parameter $\sigma_{i}$ of its $V_{i}$-indicatrix curve. Then the structure equations of the curve $\alpha$ are given by

$$
\begin{gathered}
\frac{d \alpha}{d \sigma_{i}}=\frac{1}{\sqrt{\left(\kappa_{i-1}\right)^{2}+\left(\kappa_{i}\right)^{2}}} V_{1}(s), \\
\frac{d}{d \sigma_{i}}\left(V_{1}, V_{2}, \ldots, V_{n-1}, V_{n}\right)^{T}=K\left(V_{1}, V_{2}, \ldots, V_{n-1}, V_{n}\right)^{T}
\end{gathered}
$$

where

$$
K=\left(\begin{array}{cclcc}
0 & \frac{\kappa_{1}}{\sqrt{\left(\kappa_{i-1}\right)^{2}+\left(\kappa_{i}\right)^{2}}} & \cdots & 0 & 0 \\
-\frac{\kappa_{1}}{\sqrt{\left(\kappa_{i-1}\right)^{2}+\left(\kappa_{i}\right)^{2}}} & 0 & \cdots & 0 & 0 \\
0 & -\frac{\kappa_{2}}{\sqrt{\left(\kappa_{i-1}\right)^{2}+\left(\kappa_{i}\right)^{2}}} & \cdots & 0 & 0 \\
0 & 0 & \cdots & 0 & 0 \\
\vdots & \vdots & & \vdots & \vdots \\
0 & 0 & \cdots & 0 & \frac{\kappa_{n-1}}{\sqrt{\left(\kappa_{i-1}\right)^{2}+\left(\kappa_{i}\right)^{2}}} \\
0 & 0 & \cdots & -\frac{\kappa_{n-1}}{\sqrt{\left(\kappa_{i-1}\right)^{2}+\left(\kappa_{i}\right)^{2}}} & 0
\end{array}\right)
$$


The orthogonal n-frame of the curve $\alpha$ is choosen as follows

$$
\left\{\frac{1}{\sqrt{\left(\kappa_{i-1}\right)^{2}+\left(\kappa_{i}\right)^{2}}} V_{1}(s), \frac{1}{\sqrt{\left(\kappa_{i-1}\right)^{2}+\left(\kappa_{i}\right)^{2}}} V_{2}(s), \ldots, \frac{1}{\sqrt{\left(\kappa_{i-1}\right)^{2}+\left(\kappa_{i}\right)^{2}}} V_{n}(s)\right\} .
$$

Also, if we use the following notations

$$
\tilde{\kappa}=\frac{d}{d s}\left(\frac{1}{\sqrt{\left(\kappa_{i-1}\right)^{2}+\left(\kappa_{i}\right)^{2}}}\right) \text { and } \tilde{\kappa}_{j}=\frac{\kappa_{j}}{\sqrt{\left(\kappa_{i-1}\right)^{2}+\left(\kappa_{i}\right)^{2}}}, j=1,2, \ldots, n-1 .
$$

then the derivatives of these vectors according to $\sigma_{i}$ are obtained as follows

$$
\frac{d}{d \sigma_{i}}\left(\frac{1}{\sqrt{\left(\kappa_{i-1}\right)^{2}+\left(\kappa_{i}\right)^{2}}} V_{1}, \ldots, \frac{1}{\sqrt{\left(\kappa_{i-1}\right)^{2}+\left(\kappa_{i}\right)^{2}}} V_{n}\right)^{T}=\widetilde{K}\left(\frac{1}{\sqrt{\left(\kappa_{i-1}\right)^{2}+\left(\kappa_{i}\right)^{2}}} V_{1}, \ldots, \frac{1}{\sqrt{\left(\kappa_{i-1}\right)^{2}+\left(\kappa_{i}\right)^{2}}} V_{n}\right)^{T}
$$

where

$$
\widetilde{K}=\left(\begin{array}{ccccccc}
\tilde{\kappa} & \tilde{\kappa}_{1} & 0 & \cdots & 0 & 0 & 0 \\
-\tilde{\kappa}_{1} & \tilde{\kappa} & \tilde{\kappa}_{2} & \cdots & 0 & 0 & 0 \\
0 & -\tilde{\kappa}_{2} & \tilde{\kappa} & \cdots & 0 & 0 & 0 \\
0 & 0 & -\tilde{\kappa}_{3} & \cdots & 0 & 0 & 0 \\
\vdots & \vdots & \vdots & & \vdots & \vdots & \vdots \\
0 & 0 & 0 & \cdots & -\tilde{\kappa}_{n-2} & \tilde{\kappa} & \tilde{\kappa}_{n-1} \\
0 & 0 & 0 & \cdots & 0 & -\tilde{\kappa}_{n-1} & \tilde{\kappa}
\end{array}\right) .
$$

Remark 3.1. For $i=1$ (that is, $\sigma_{i}=\sigma$ ) the Eq. (3.3) coincidence with Eq. (2.6) in [2].

Definition 3.1. Let $\alpha: I \subset \mathbb{R} \rightarrow E^{n}$ be a Frenet curve parameterized by an arc length parameter $\sigma_{i}$ of its $V_{i}-$ indicatrix curve. The functions

$$
\tilde{\kappa}\left(\sigma_{i}\right)=-\frac{1}{\sqrt{\left(\kappa_{i-1}\right)^{2}+\left(\kappa_{i}\right)^{2}}} \frac{d\left(\sqrt{\left(\kappa_{i-1}\right)^{2}+\left(\kappa_{i}\right)^{2}}\right)}{d \sigma_{i}} \text { and } \tilde{\kappa}_{j}\left(\sigma_{i}\right)=\frac{\kappa_{j}}{\sqrt{\left(\kappa_{i-1}\right)^{2}+\left(\kappa_{i}\right)^{2}}}, j=1,2, \ldots, n-1
$$

are called shape curvatures of the curve $\alpha$.

Proposition 3.1. Let $\alpha\left(\sigma_{i}\right): I \subset \mathbb{R} \rightarrow \mathbb{E}^{n}$ be a Frenet curve with the orthogonal $n$-frame

$$
\left\{\frac{1}{\sqrt{\left(\kappa_{i-1}\right)^{2}+\left(\kappa_{i}\right)^{2}}} V_{1}, \frac{1}{\sqrt{\left(\kappa_{i-1}\right)^{2}+\left(\kappa_{i}\right)^{2}}} V_{2}, \ldots, \frac{1}{\sqrt{\left(\kappa_{i-1}\right)^{2}+\left(\kappa_{i}\right)^{2}}} V_{n}\right\} .
$$

The shape curvatures of the curve $\alpha$

$$
\tilde{\kappa}\left(\sigma_{i}\right)=-\frac{1}{\sqrt{\left(\kappa_{i-1}\right)^{2}+\left(\kappa_{i}\right)^{2}}} \frac{d\left(\sqrt{\left(\kappa_{i-1}\right)^{2}+\left(\kappa_{i}\right)^{2}}\right)}{d \sigma_{i}} \text { and } \tilde{\kappa}_{j}\left(\sigma_{i}\right)=\frac{\kappa_{j}}{\sqrt{\left(\kappa_{i-1}\right)^{2}+\left(\kappa_{i}\right)^{2}}}, j=1,2, \ldots, n-1
$$

are differential geometric invariants determining the curve $\alpha$ up to a direct similarity.

Proof. Let $\left\{\bar{V}_{1}, \bar{V}_{2}, \ldots, \bar{V}_{n}, \bar{\kappa}_{1}, \bar{\kappa}_{2}, \ldots, \bar{\kappa}_{(n-1)}\right\}$ be a Frenet apparatus of the curve $\bar{\alpha}=F \circ \alpha$. The shape curvatures of the curve $\bar{\alpha}$ are given by

$$
\widetilde{\widetilde{\kappa}}\left(\sigma_{i}\right)=-\frac{1}{\sqrt{\left(\bar{\kappa}_{i-1}\right)^{2}+\left(\bar{\kappa}_{i}\right)^{2}}} \frac{d\left(\sqrt{\left(\bar{\kappa}_{i-1}\right)^{2}+\left(\bar{\kappa}_{i}\right)^{2}}\right)}{d \bar{\sigma}_{i}}
$$


By using the $\bar{\kappa}_{i}=\frac{1}{\lambda} \kappa_{i}(s), i=1,2, \ldots, n-1$ and $\frac{d s}{d \bar{s}}=\frac{1}{\lambda}$, we have

$$
\widetilde{\bar{\kappa}}\left(\sigma_{i}\right)=\tilde{\kappa}\left(\sigma_{i}\right)
$$

and we obtain

$$
\begin{aligned}
\widetilde{\kappa}_{j}\left(\sigma_{i}\right) & =\frac{\bar{\kappa}_{j}}{\sqrt{\left(\bar{\kappa}_{i-1}\right)^{2}+\left(\bar{\kappa}_{i}\right)^{2}}}, \\
\widetilde{\kappa}_{j}\left(\sigma_{i}\right) & =\tilde{\kappa}_{j}\left(\sigma_{i}\right) .
\end{aligned}
$$

So, the desired results are obtained.

Remark 3.2. For $i=1$ (that is, $\left.\sigma_{i}=\sigma\right) \tilde{\kappa}$ and $\tilde{\kappa}_{j}(j=2, \ldots, n-1)$ coincidence with $\tilde{\kappa}_{1}$ and $\tilde{\kappa}_{i}(i=2,3, \ldots, n-1)$ respectively in [2].

\section{The relation between the curve $\alpha$ and its evolute curve}

Definition 4.1. Let $\alpha: I \subset \mathbb{R} \rightarrow E^{3}$ be a unit speed Frenet curve with Frenet apparatus $\left\{\kappa_{1}, \kappa_{2}, V_{1}, V_{2}, V_{3}\right\}$ and $\beta$ be an evolute curve of the curve $\alpha$. Then there exist the following equality

$$
\beta(s)=\alpha(s)+m_{1}(s) V_{2}(s)+m_{2}(s) V_{3}(s)
$$

where $m_{1}(s)=\frac{1}{\kappa_{1}(s)}, m_{2}(s)=\frac{1}{\kappa_{1}(s)} \cot \left(\int \kappa_{2}(s) d s\right)$ [4].

In [6], R. Uribe-Vargas found formulas which express the Euclidean curvatures in terms of the focal curvatures. Similarly, we can represent all differential-geometric invariants $\tilde{\kappa}_{i}$ by the $m_{i}(i=1,2)$ curvatures and their derivatives. For $i=1$, we have $\tilde{\kappa}_{1}=\frac{d}{d s}\left(\frac{1}{\kappa_{1}}\right)$ and $\tilde{\kappa}_{2}=\frac{\kappa_{2}}{\kappa_{1}}$.

Proposition 4.1. Let $\alpha: I \rightarrow \mathbb{R}^{3}$ be a unit speed Frenet curve. Then, the shape curvatures $\tilde{\kappa}_{i}(i=1,2)$ of the curve $\alpha$ are given in terms of the curvatures $m_{i}$ following as

$$
\tilde{\kappa}_{1}=m_{1}^{\prime} \quad \text { and } \quad \tilde{\kappa}_{2}=\frac{m_{1}\left(m_{1}^{\prime} m_{2}-m_{1} m_{2}^{\prime}\right)}{m_{1}^{2}+m_{2}^{2}} .
$$

Proof. Since $\tilde{\kappa}_{1}=\frac{d}{d s}\left(\frac{1}{\kappa_{1}}\right)$ and $\tilde{\kappa}_{2}=\frac{\kappa_{2}}{\kappa_{1}}$ then we get

$$
\begin{gathered}
m_{1}^{\prime}(s)=\left(\frac{1}{\kappa_{1}(s)}\right)^{\prime}=\tilde{\kappa}_{1} . \\
m_{2}^{\prime}(s)=\left(\frac{1}{\kappa_{1}(s)} \cot \left(\int \kappa_{2}(s) d s\right)\right)^{\prime} \\
=m_{1}^{\prime} \frac{m_{2}}{m_{1}}-\frac{\kappa_{2}}{\kappa_{1}} \frac{1}{\sin ^{2}\left(\int \kappa_{2}(s) d s\right)} \\
\tilde{\kappa}_{2}=\frac{m_{1}\left(m_{1}^{\prime} m_{2}-m_{1} m_{2}^{\prime}\right)}{m_{1}^{2}+m_{2}^{2}} .
\end{gathered}
$$

\section{The relation between the curve $\alpha$ and its focal curve}

Let $\alpha: I \subset \mathbb{R} \rightarrow E^{n}$ be a unit speed Frenet curve. Suppose that all Euclidean curvatures of the curve $\alpha$ are nonzero for any $s \in I$. The curve $C_{\alpha}: I \subset \mathbb{R} \rightarrow E^{n}$ consisting of the centers of the osculating spheres of the curve $\alpha$ is called the focal curve of the curve $\alpha$. Then the focal curve $C_{\alpha}$ has a representation:

$$
C_{\alpha}=\alpha(s)+f_{1}(s) V_{2}(s)+\ldots+f_{n-2}(s) V_{n-1}(s)+f_{n-1}(s) V_{n}(s)
$$

where the functions $f_{i}(s), i=1, \ldots, n-1$ are called focal curvatures of the curve $\alpha$. In [6], Uribe-Vargas found an equation between the Euclidean curvatures and the focal curvatures. Then by using this equation, we can express the shape curvatures of the curve $\alpha$ in terms of focal curvatures. 
Proposition 5.1. Let $\alpha: I \subset \mathbb{R} \rightarrow E^{n}$ be a space curve with all Euclidean curvatures different from zero. Then we have

$$
\begin{gathered}
\tilde{\kappa}=\frac{d}{d s}\left(\frac{f_{i-2} f_{i-1} f_{i}}{\sqrt{\left(\left(f_{1} f_{1}^{\prime}+f_{2} f_{2}^{\prime}+\cdots+f_{i-2} f_{i-2}^{\prime}\right) f_{i}\right)^{2}+\left(\left(f_{1} f_{1}^{\prime}+f_{2} f_{2}^{\prime}+\cdots+f_{i-1} f_{i-1}^{\prime}\right) f_{i-2}\right)^{2}}}\right), \\
\tilde{\kappa}_{j}=\frac{f_{1-2} f_{i-1}^{\prime} f_{i}}{f_{j-1} f_{j}} \frac{f_{2} f_{2}^{\prime}+\cdots+f_{j-1} f_{j-1}^{\prime}}{\sqrt{\left(\left(f_{1} f_{1}^{\prime}+f_{2} f_{2}^{\prime}+\cdots+f_{i-2} f_{i-2}^{\prime}\right) f_{i}\right)^{2}+\left(\left(f_{1} f_{1}^{\prime}+f_{2} f_{2}^{\prime}+\cdots+f_{i-1} f_{i-1}^{\prime}\right) f_{i-2}\right)^{2}}}, \\
j=1, \ldots, n-1 .
\end{gathered}
$$

Proof. According to the first two theorems in [6], there is a relation between the Frenet curvatures and the focal curvatures as follows:

$$
\kappa_{1}=\frac{1}{f_{1}} \text { and } \kappa_{i}=\frac{f_{1} f_{1}^{\prime}+f_{2} f_{2}^{\prime}+\cdots+f_{i-1} f_{i-1}^{\prime}}{f_{i-1} f_{i}}, \quad i=2,3, \ldots, n-1 .
$$

By using the Eq. (3.5), we get the Eq. (5.3).

Remark 5.1. For $i=1$ (that is $\sigma_{i}=\sigma$ ), the representation of the shape curvatures is given with Eq.(5.2) the same as representation of the shape curvatures is given with Eq. (4.2) in [2].

\section{Self-Similar Frenet Curves}

The curve $\alpha: I \subset \mathbb{R} \rightarrow E^{n}$ is called a self-similar curve if and only if all its invariants $\tilde{\kappa}, \tilde{\kappa}_{1}, \ldots, \tilde{\kappa}_{n-1}$ are constant.

$$
K=\left(\begin{array}{cccccccc}
0 & \tilde{\kappa}_{1} & 0 & 0 & \cdots & 0 & 0 & 0 \\
-\tilde{\kappa}_{1} & 0 & \tilde{\kappa}_{2} & 0 & \cdots & 0 & 0 & 0 \\
0 & -\tilde{\kappa}_{2} & 0 & \tilde{\kappa}_{3} & \cdots & 0 & 0 & 0 \\
\vdots & \vdots & \vdots & \vdots & \ddots & \vdots & \vdots & \vdots \\
0 & 0 & 0 & 0 & \cdots & -\tilde{\kappa}_{n-2} & 0 & \tilde{\kappa}_{n-1} \\
0 & 0 & 0 & 0 & \cdots & 0 & -\tilde{\kappa}_{n-1} & 0
\end{array}\right) .
$$

According to [3], the normal form of the matrix $K$ is given by

$$
\left(\begin{array}{cccccccc}
0 & \lambda_{1} & 0 & 0 & \cdots & 0 & 0 & 0 \\
-\lambda_{1} & 0 & 0 & 0 & \cdots & 0 & 0 & 0 \\
0 & 0 & 0 & \lambda_{2} & \cdots & 0 & 0 & 0 \\
0 & 0 & -\lambda_{2} & 0 & \cdots & 0 & 0 & 0 \\
\vdots & \vdots & \vdots & \vdots & \ddots & \vdots & \vdots & \vdots \\
0 & 0 & 0 & 0 & \cdots & 0 & 0 & \lambda_{m} \\
0 & 0 & 0 & 0 & \cdots & 0 & -\lambda_{m} & 0
\end{array}\right) .
$$

Then the symmetric matrix $K^{2}$ has $m=\frac{n}{2}$ negative eigenvalues with multiplicity two: $-\lambda_{1}^{2},-\lambda_{2}^{2}, \ldots,-\lambda_{m}^{2}$.

\section{Self- Similar Curves in Even-Dimensional Euclidean Spaces}

In this section, we get the expression of the self-similar curve $\alpha$ according to the parameter $\sigma_{i}$ in the even dimensional real space $\mathbb{R}^{2 m}$.

Theorem 6.1. Let $\alpha: I \rightarrow \mathbb{R}^{2 m}$ be a self-similar Frenet curve. The curve $\alpha$, parameterized by the arc length parameter $\sigma_{i}$ of its $V_{i}$-indicatrix curve, is given by

$$
\alpha\left(\sigma_{i}\right)=\left(\frac{a_{1}}{b_{1}} e^{\tilde{\kappa} \sigma_{i}} \sin \theta_{1},-\frac{a_{1}}{b_{1}} e^{\tilde{\kappa} \sigma_{i}} \cos \theta_{1}, \cdots, \frac{a_{m}}{b_{m}} e^{\tilde{\kappa} \sigma_{i}} \sin \theta_{m},-\frac{a_{m}}{b_{m}} e^{\tilde{\kappa} \sigma_{i}} \cos \theta_{m}\right)
$$


where $b_{j}=\sqrt{\tilde{\kappa}^{2}+\lambda_{j}^{2}}$ and $\theta_{j}=\sqrt{\lambda_{j}^{2} \sigma_{i}}+\arccos \left(\frac{\sqrt{\lambda_{j}^{2}}}{\sqrt{\tilde{\kappa}^{2}+\lambda_{j}^{2}}}\right)$ for $j=1,2, \ldots, m$. The real different nonzero numbers $a_{1}, a_{2}, \cdots, a_{m}$ are solution of the system

$$
\left\langle V_{j}, V_{j}\right\rangle=1, \quad 1 \leqslant j \leqslant m
$$

Proof. The unit vector fields $\left\{V_{1}\left(\sigma_{i}\right), V_{2}\left(\sigma_{i}\right), \cdots, V_{2 m-1}\left(\sigma_{i}\right), V_{2 m}\left(\sigma_{i}\right)\right\}$ are expressed in the form as follows:

$$
\omega\left(\sigma_{i}\right)=\left(V_{1}\left(\sigma_{i}\right), V_{2}\left(\sigma_{i}\right), \cdots, V_{2 m-1}\left(\sigma_{i}\right), V_{2 m}\left(\sigma_{i}\right)\right) .
$$

From the solution of the differential equation $\frac{d}{d \sigma_{i}} \omega=K \omega$, we can calculate the unit vector fields

$$
\left\{V_{1}\left(\sigma_{i}\right), V_{2}\left(\sigma_{i}\right), \cdots, V_{2 m-1}\left(\sigma_{i}\right), V_{2 m}\left(\sigma_{i}\right)\right\}
$$

Also, the first unit vector is calculated by

$$
V_{1}\left(\sigma_{i}\right)=\left(a_{1} \cos \left(\lambda_{1} \sigma_{i}\right), a_{1} \sin \left(\lambda_{1} \sigma_{i}\right), \cdots a_{m} \cos \left(\lambda_{m} \sigma_{i}\right), a_{m} \sin \left(\lambda_{m} \sigma_{i}\right)\right) .
$$

Since $\left\langle V_{1}, V_{1}\right\rangle=1$, we obtain that $\sum_{j=1}^{m} a_{j}^{2}=1$. The parametric equation of the curve $\alpha$ is given by

$$
X=\left(x_{1}(\sigma), x_{2}(\sigma), \cdots, x_{2 m-1}(\sigma), x_{2 m}(\sigma)\right) .
$$

By using the Eq. (3.1), we can write $\frac{d}{d \sigma_{i}} X=\frac{1}{\sqrt{\kappa_{i-1}^{2}+\kappa_{i}^{2}}} V_{1}$. We can easily see that $\sqrt{\kappa_{i-1}^{2}+\kappa_{i}^{2}}=e^{-\tilde{\kappa} \sigma_{i}}$. Hence, we have

$$
\frac{d}{d \sigma_{i}} x_{2 j-1}=a_{j} e^{\tilde{\kappa} \sigma} \cos \left(\lambda_{j} \sigma_{i}\right) \quad \text { and } \quad \frac{d}{d \sigma_{i}} x_{2 j}=a_{j} e^{\tilde{\kappa} \sigma_{i}} \sin \left(\lambda_{j} \sigma_{i}\right), \quad 1 \leqslant j \leqslant m .
$$

Taking the integrals of the last equations, we obtain

$$
\begin{aligned}
& x_{2 j-1}=\frac{a_{j}}{\tilde{\kappa}} e^{\tilde{\kappa}_{1} \sigma_{i}} \cos \left(\lambda_{j} \sigma_{i}\right)+\frac{\lambda_{j}}{\tilde{\kappa}} x_{2 j}, \\
& x_{2 j}=\frac{a_{j}}{\tilde{\kappa}} e^{\tilde{\kappa}_{1} \sigma_{i}} \sin \left(\lambda_{j} \sigma_{i}\right)-\frac{\lambda_{i}}{\tilde{\kappa}} x_{2 j-1} .
\end{aligned}
$$

Using the Eq.(6.2) and Eq.(6.3), we get two equations:

$$
\begin{aligned}
x_{2 j-1} & =\frac{a_{j}}{\tilde{\kappa}^{2}+\lambda_{j}^{2}} e^{\tilde{\kappa} \sigma_{i}}\left(\tilde{\kappa} \cos \left(\lambda_{j} \sigma_{i}\right)+\lambda_{j} \sin \left(\lambda_{j} \sigma_{i}\right)\right)=\frac{a_{j}}{b_{j}} e^{\tilde{\kappa} \sigma_{i}} \sin \theta_{j}, \\
x_{2 j} & =\frac{a_{j}}{\tilde{\kappa}^{2}+\lambda_{j}^{2}} e^{\tilde{\kappa} \sigma_{i}}\left(\tilde{\kappa} \sin \left(\lambda_{j} \sigma_{i}\right)-\lambda_{j} \cos \left(\lambda_{j} \sigma_{i}\right)\right)=-\frac{a_{j}}{b_{j}} e^{\tilde{\kappa} \sigma_{i}} \cos \theta_{j}
\end{aligned}
$$

where

$$
\begin{aligned}
\sin \theta_{j} & =\frac{b_{j}}{\tilde{\kappa}}\left(\cos \left(\lambda_{j} \sigma_{i}\right)-\frac{\lambda_{j}}{b_{j}} \cos \theta_{j}\right), \\
\cos \theta_{j} & =-\frac{b_{j}}{\tilde{\kappa}}\left(\sin \left(\lambda_{j} \sigma_{i}\right)-\frac{\lambda_{j}}{b_{j}} \sin \theta_{j}\right) .
\end{aligned}
$$

So, the function $\theta_{j}$ is found

$$
\theta_{j}=\lambda_{j} \sigma_{i}+\arccos \frac{\lambda_{j}}{\sqrt{\tilde{\kappa}^{2}+\lambda_{j}^{2}}}
$$


We can write from the equation $\frac{d}{d \sigma_{i}} \omega=K \omega$

$$
\begin{aligned}
V_{1}\left(\sigma_{i}\right)= & e^{-\tilde{\kappa} \sigma_{i}} \frac{d}{d \sigma_{i}} \alpha\left(\sigma_{i}\right), \\
V_{2}\left(\sigma_{i}\right)= & \frac{1}{\tilde{\kappa}_{1}} \frac{d}{d \sigma_{i}} V_{1}\left(\sigma_{i}\right), \\
V_{3}\left(\sigma_{i}\right)= & \frac{1}{\tilde{\kappa}_{2}}\left(-\tilde{k}_{1} V_{1}\left(\sigma_{i}\right)+\frac{d}{d \sigma_{i}} V_{2}\left(\sigma_{i}\right)\right), \\
V_{4}\left(\sigma_{i}\right)= & \frac{1}{\tilde{\kappa}_{3}}\left(\tilde{\kappa}_{2} V_{2}\left(\sigma_{i}\right)+\frac{d}{d \sigma_{i}} V_{3}\left(\sigma_{i}\right)\right), \\
& \vdots \\
V_{m}\left(\sigma_{i}\right)= & \frac{1}{\tilde{\kappa}_{m-1}}\left(\tilde{\kappa}_{m-2} V_{m-2}\left(\sigma_{i}\right)+\frac{d}{d \sigma_{i}} V_{m-1}\left(\sigma_{i}\right)\right) .
\end{aligned}
$$

By using an algebric calculus, we obtain the following equations:

$$
\begin{aligned}
\left\langle V_{1}, V_{1}\right\rangle & =1 \Rightarrow \sum_{j=1}^{m} a_{j}^{2}=1, \\
\left\langle V_{2}, V_{2}\right\rangle & =1 \Rightarrow \sum_{j=1}^{m} a_{j}^{2} \lambda_{j}^{2}=\tilde{\kappa}_{1}^{2}, \\
\left\langle V_{3}, V_{3}\right\rangle & =1 \Rightarrow \sum_{j=1}^{m} a_{j}^{2}\left(1-\lambda_{j}^{2}\right)^{2}=\tilde{\kappa}_{2}^{2}, \\
\left\langle V_{4}, V_{4}\right\rangle & =1 \Rightarrow \sum_{j=1}^{m} a_{j}^{2} \lambda_{j}^{2}\left(\tilde{\kappa}_{2}^{2}-\lambda_{j}^{2}\right)^{2}=\tilde{\kappa}_{2}^{2} \tilde{\kappa}_{3}^{2},
\end{aligned}
$$

and so on. The proof is completed.

Remark 6.1. In the Eq.(6.1), if we take the $\sigma_{i}=\sigma$, then we obtain the Eq. (5.2) in [2].

\section{Self-Similar Curves in Odd-Dimensional Euclidean Spaces}

In this section, we give also express the self-similar curves in odd-dimensional Euclidean space $\mathbb{R}^{2 m+1}$.

Theorem 6.2. Let $\alpha: I \rightarrow \mathbb{R}^{2 m+1}$ be a self-similar curve. The curve $\alpha$, parameterized by the arc length parameter $\sigma_{i}$ of its $V_{i}$-indicatrix curve, is given by

$$
\alpha\left(\sigma_{i}\right)=\left(\frac{a_{1}}{b_{1}} e^{\tilde{\kappa} \sigma_{i}} \sin \theta_{1},-\frac{a_{1}}{b_{1}} e^{\tilde{\kappa} \sigma_{i}} \cos \theta_{1}, \cdots, \frac{a_{m}}{b_{m}} e^{\tilde{\kappa} \sigma_{i}} \sin \theta_{m},-\frac{a_{m}}{b_{m}} e^{\tilde{\kappa} \sigma_{i}} \cos \theta_{m}, a_{m+1} e^{\tilde{\kappa} \sigma_{i}}\right)
$$

where $b_{j}=\sqrt{\tilde{\kappa}^{2}+\lambda_{j}^{2}}$ and $\theta_{j}=\sqrt{\lambda_{j}^{2} \sigma_{i}}+\arccos \left(\frac{\sqrt{\lambda_{j}^{2}}}{\sqrt{\tilde{\kappa}^{2}+\lambda_{j}^{2}}}\right)$ for $1 \leqslant j \leqslant m$. To calculate the real different nonzero numbers $a_{1}, a_{2}, \cdots, a_{m+1}$, we use the following $(m+1)$ - equations

$$
\begin{aligned}
V_{1}\left(\sigma_{i}\right)= & e^{-\tilde{\kappa} \sigma_{i}} \frac{d}{d \sigma_{i}}, \alpha\left(\sigma_{i}\right) \\
V_{2}\left(\sigma_{i}\right)= & \frac{1}{\tilde{\kappa}_{1}} \frac{d}{d \sigma_{i}} V_{1}\left(\sigma_{i}\right), \\
V_{3}\left(\sigma_{i}\right)= & \frac{1}{\tilde{\kappa}_{2}}\left(-\tilde{\kappa}_{1} V_{1}\left(\sigma_{i}\right)+\frac{d}{d \sigma_{i}} V_{2}\left(\sigma_{i}\right)\right), \\
V_{4}\left(\sigma_{i}\right)= & \frac{1}{\tilde{\kappa}_{3}}\left(\tilde{\kappa}_{2} V_{2}\left(\sigma_{i}\right)+\frac{d}{d \sigma_{i}} V_{3}\left(\sigma_{i}\right)\right), \\
& \vdots \\
V_{m+1}\left(\sigma_{i}\right)= & \frac{1}{\tilde{\kappa}_{m}}\left(\tilde{\kappa}_{m-1} V_{m-1}\left(\sigma_{i}\right)+\frac{d}{d \sigma_{i}} V_{m}\left(\sigma_{i}\right)\right) .
\end{aligned}
$$




$$
\left\langle V_{j}, V_{j}\right\rangle=1, \quad 1 \leqslant j \leqslant m+1 .
$$

From the above statements, we obtain the following equalities

$$
\begin{aligned}
\left\langle V_{1}, V_{1}\right\rangle & =1 \Rightarrow \sum_{i=1}^{m} a_{i}^{2}+\tilde{\kappa}^{2} a_{m+1}^{2}=1 \\
\left\langle V_{2}, V_{2}\right\rangle & =1 \Rightarrow \sum_{i=1}^{m+1} a_{i}^{2} \lambda_{i}^{2}=\tilde{\kappa}_{1}^{2} \\
\left\langle V_{3}, V_{3}\right\rangle & =1 \Rightarrow \sum_{i=1}^{m} a_{i}^{2}\left(1-\frac{\lambda_{i}^{2}}{\tilde{\kappa}_{1}^{2}}\right)^{2}+\tilde{\kappa}^{2} a_{m+1}^{2}=\frac{\tilde{\kappa}_{2}^{2}}{\tilde{\kappa}_{1}^{2}} \\
\left\langle V_{i}, V_{i}\right\rangle & =1,4 \leqslant i \leqslant m+1 .
\end{aligned}
$$

Proof. The proof can be made similar to the proof of Theorem 6.1.

Remark 6.2. For $i=1$ (that is $\sigma_{i}=\sigma$ ), the representation of the curve $\alpha$ in the Eq.(6.4) is the same as the curve given in the Eq. (5.4) in [2].

Example 6.1. The shape curvature functions $\tilde{\kappa}_{1}=\frac{3}{\sqrt{13}}$ and $\tilde{\kappa}_{2}=\frac{2}{\sqrt{13}}$ of the curve $\alpha$ are in $\mathbb{R}^{3}$. We can calculate the curve $\alpha$ (see Figure 1) corresponding to them

$$
\alpha\left(\sigma_{2}\right)=\left(\frac{a_{1}}{b_{1}} e^{\tilde{\kappa} \sigma_{2}} \sin \theta_{1},-\frac{a_{1}}{b_{1}} e^{\tilde{\kappa} \sigma_{2}} \cos \theta_{1}, a_{2} e^{\tilde{\kappa} \sigma_{2}}\right)
$$

where $\sigma_{2}$ is arc length parameter of its normal indicatrix curve. If we use the given equations in Theorem 6.2 for $m=1$, we get the values needed to determine the curve as follows:

$$
\lambda_{1}^{2}=\frac{5}{13}, a_{1}=\frac{3}{\sqrt{5}} \text { and } a_{2}=\frac{2}{\sqrt{5}}, b_{1}=\frac{\sqrt{36+5 s^{4}}}{\sqrt{13} s^{2}}, \theta_{1}=\sqrt{\frac{5}{13}} \sigma_{2}+\arccos \left(\frac{\sqrt{5}}{\sqrt{\frac{36}{s^{4}}+5}}\right) .
$$

The curve $\alpha$ for these values is plotted using the Mathematica program in figure 1 .

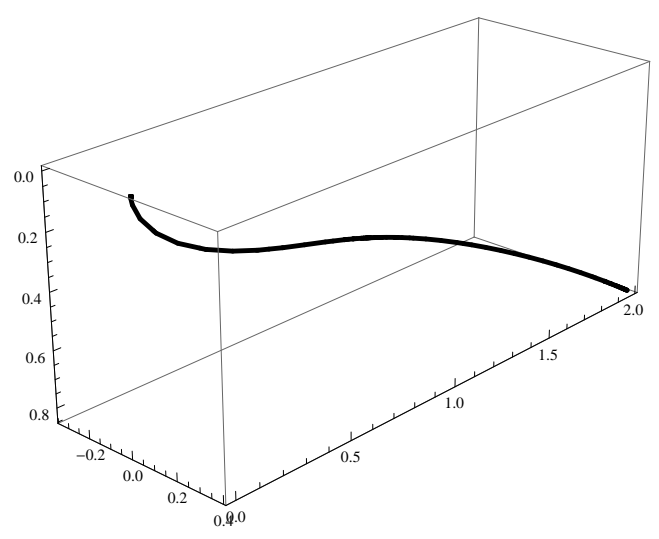

Figure 1. The curve $\alpha$. 


\section{Geometric Interpretation of the Shape Curvatures in Euclidean 3-Space}

In this section, we investigate that the shape curvatures of the curve are related to the geodesic curvatures of its indicatrix curves.

Proposition 7.1. Geodesic curvatures of indicatrix curves are invariant under the group $\operatorname{Sim}^{+}\left(\mathbb{R}^{3}\right)$.

Proof. Let $\alpha: I \rightarrow \mathbb{R}^{3}$ be a Frenet curve with Frenet apparatus $\left\{V_{1}, V_{2}, V_{3}, \kappa_{1}, \kappa_{2}\right\}$ and $\tilde{\kappa}_{1}, \tilde{\kappa}_{2}$ be shape curvatures. Its indicatrix curve $\gamma: I \rightarrow S^{2}$ is a spherical curve with arc length parameter $\sigma_{i}(i=1,2,3)$. The vector $t\left(\sigma_{i}\right)=\frac{d}{d \sigma_{i}} \gamma\left(\sigma_{i}\right)$ is a unit tangent vector of the curve $\gamma$. Third vector $\rho\left(\sigma_{i}\right)=\gamma\left(\sigma_{i}\right) \wedge t\left(\sigma_{i}\right)$ is defined along the curve $\gamma$ on the unit sphere. This frame is called the Sabban frame of the curve $\gamma$ on the unit sphere $S^{2}$. Also, the derivetives of the vectors of the Sabban frame are defined as follows:

$$
\frac{d}{d \sigma_{i}}\left[\begin{array}{l}
\gamma \\
t \\
\rho
\end{array}\right]=\left[\begin{array}{ccc}
0 & 1 & 0 \\
-1 & 0 & \kappa_{g} \\
0 & -\kappa_{g} & 0
\end{array}\right]\left[\begin{array}{l}
\gamma \\
t \\
\rho
\end{array}\right]
$$

where the function $\kappa_{g}=\operatorname{det}\left(\gamma, t, \frac{d t}{d \sigma_{i}}\right)$ is called the geodesic curvature of the curve $\gamma$.

Let $\bar{\alpha}$ be a similar curve of the curve $\alpha$ under the group $\operatorname{Sim}^{+}\left(\mathbb{R}^{3}\right)$ and $\left\{\bar{V}_{1}, \bar{V}_{2}, \bar{V}_{3}, \bar{\kappa}_{1}, \bar{\kappa}_{2}, \widetilde{\kappa}_{1}, \widetilde{\bar{\kappa}}_{2}\right\}$ be Frenet apparatus of the curve $\bar{\alpha}$. Its indicatrix curve $\bar{\gamma}: I \rightarrow S^{2}$ is a spherical curve with arc length parameter $\bar{\sigma}_{i}(i=1,2,3)$. The orthogonal frame $\left\{\bar{\gamma}\left(\bar{\sigma}_{i}\right), \bar{t}\left(\bar{\sigma}_{i}\right), \bar{\rho}\left(\bar{\sigma}_{i}\right)\right\}$ along the curve $\bar{\gamma}$ is called the Sabban frame on the unit sphere.

If the curve $\gamma$ is a tangent indicatrix curve with arc length parameter $\sigma_{1}$ then the following equalities hold

$$
\begin{gathered}
t\left(\sigma_{1}\right)=\frac{d}{d \sigma_{1}} \gamma\left(\sigma_{1}\right), \\
\frac{d}{d \sigma_{1}} t\left(\sigma_{1}\right)=-\gamma\left(\sigma_{1}\right)+\frac{\tilde{\kappa}_{2}}{\tilde{\kappa}_{1}} \rho\left(\sigma_{1}\right) .
\end{gathered}
$$

So, the geodesic curvature $\kappa_{g}=\frac{\tilde{\kappa}_{2}}{\tilde{\kappa}_{1}}$ is obtained. Similarly, we calculate $\bar{\kappa}_{g}=\frac{\widetilde{\widetilde{\kappa}}_{2}}{\widetilde{\widetilde{\kappa}}_{1}}$. From the Proposition 3.1, we get $\bar{\kappa}_{g}=\kappa_{g}$.

If the curve $\gamma$ is a normal indicatrix curve with arc length parameter $\sigma_{2}$, then we obtain the following formulas

$$
\begin{aligned}
t\left(\sigma_{2}\right) & =\frac{d}{d \sigma_{2}} \gamma\left(\sigma_{2}\right), \\
\frac{d}{d \sigma_{2}} t\left(\sigma_{2}\right) & =-\gamma\left(\sigma_{2}\right)+\tilde{\kappa}_{1}^{2} \frac{d}{d \sigma_{2}}\left(\frac{\tilde{\kappa}_{2}}{\tilde{\kappa}_{1}}\right) \rho\left(\sigma_{2}\right) .
\end{aligned}
$$

From the last equation, we can easily see that $\kappa_{g}\left(\sigma_{2}\right)=\tilde{\kappa}_{1}^{2} \frac{d}{d \sigma_{2}}\left(\frac{\tilde{\kappa}_{2}}{\tilde{\kappa}_{1}}\right)$. Similarly, we get $\bar{\kappa}_{g}=\widetilde{\widetilde{\kappa}}_{1}^{2} \frac{d}{d \bar{\sigma}_{2}}\left(\frac{\widetilde{\kappa}_{2}}{\widetilde{\kappa}_{1}}\right)$. So, $\bar{\kappa}_{g}$ is equal to $\kappa_{g}$.

If the curve $\gamma$ is a binormal indicatrix curve with arc length parameter $\sigma_{3}$, then the following formulas hold

$$
\begin{aligned}
t\left(\sigma_{3}\right) & =\frac{d}{d \sigma_{3}} \gamma\left(\sigma_{3}\right), \\
\frac{d}{d \sigma_{3}} t\left(\sigma_{3}\right) & =-\gamma\left(\sigma_{3}\right)+\frac{\tilde{\kappa}_{1}}{\tilde{\kappa}_{2}} \rho\left(\sigma_{2}\right)
\end{aligned}
$$

Since $\kappa_{g}\left(\sigma_{3}\right)=\frac{\tilde{\kappa}_{1}}{\tilde{\kappa}_{2}}$ and $\bar{\kappa}_{g}=\frac{\widetilde{\kappa}_{1}}{\widetilde{\bar{\kappa}}_{2}}$ are obtained, $\kappa_{g}$ equals to $\bar{\kappa}_{g}$. 


\section{References}

[1] Encheva, R., Georgiev, G., Shapes of space curves. J. Geom. Graph 7 (2003), 145-155.

[2] Encheva, R., Georgiev, G., Georgi, H., Similar Frenet curves. Results Math. 55 (2009), no. 34, 359-372.

[3] Greub W., Linear Algebra. Springer Verlag, Heidelberg, 1967.

[4] Millman, Richard S., Parker George D., Elements of differential geometry. Prentice Hall, 1977.

[5] Struik, D. J., Lectures on Classical Differential Geometry. Dover, New York, 1988.

[6] Uribe Vargas, R., On vertices focal curvatures and differential geometry of space curves. Bull. Braz. Math. Soc. 36 (2005), no. 3, 285-307.

\section{Affiliations}

FATMA ATEŞ

AdDress: Ankara University, Faculty of Science, Department of Mathematics, 06100, Ankara-Turkey. E-MAIL: fgokcelik@ankara.edu.tr

SEHER KAYA

AdDRESS: Ankara University, Faculty of Science, Department of Mathematics, 06100, Ankara-Turkey. E-MAIL: seherkaya@ankara.edu.tr

YUSUF YAYLI

AdDRess: Ankara University, Faculty of Science, Department of Mathematics, 06100, Ankara-Turkey. E-MAIL: yayli@science.ankara.edu.tr

F. NEJAT EKMEKCI

AdDRESS: Ankara University, Faculty of Science, Department of Mathematics, 06100, Ankara-Turkey. E-MAIL: ekmekci@science.ankara.edu.tr 